December 9, 2019 3:30 WSPC/INSTRUCTION FILE～HoCL-CNYang85

\title{
Prepotential approach to exact and quasi-exact solvabilities of Hermitian and non-Hermitian Hamiltonians
}

\author{
Choon-Lin Ho \\ Department of Physics, Tamkang University, Tamsui 251, Taiwan, Republic of China
}

\begin{abstract}
In this talk I present a simple and unified approach to both exact and quasi-exact solvabilities of the one-dimensional Schrödinger equation. It is based on the prepotential together with Bethe ansatz equations. This approach gives the potential as well as the eigenfunctions and eigenvalues simultaneously. In this approach the system is completely defined by the choice of the change of variables, and the so-called zero-th order prepotential. We illustrate the approach by several examples of Hermitian and non-Hermitian Hamiltonians with real energies. The method can be easily extended to the constructions of exactly and quasi-exactly solvable Dirac, Pauli, and Fokker-Planck equations, and to quasinormal modes.
\end{abstract}

Keywords: Prepotential, exact/quasi-exact solvability, Bethe-ansatz equations, nonHermitian Hamiltonians

\section{Introduction}

I am greatly honored to be able to participate in this conference celebrating Prof. Yang's 85th birthday. We wish Prof. Yang a very happy birthday, and many more to come.

In this talk I would like to present a constructive approach to both exact and quasi-exact solvable one-dimensional Schrödinger equations. Everyone knows what exact solvability means. It is fair to say that all of us learned the principles of quantum mechanics through several well-known exactly solvable (ES) models, such as the infinite square well, the harmonic oscillator, the hydrogen atom, etc. But in actual life, exactly solvable systems are hard to come by. Most systems one encounters are non-solvable, and one has to adopt various approximation schemes to solve them.

Two decades ago, a new class of potentials which are intermediate to ES potentials and non solvable ones have been found for the Schrödinger equation. These are called quasi-exactly solvable (QES) models for which it is possible to determine algebraically a part of the spectrum but not the whole spectrum $1|2| 3|4| 5 \mid 6$. The simplest QES model first discovered is the sextic oscillator 1 . The discovery of this class of spectral problems has greatly enlarged the number of physical systems which we can study analytically.

Here I would like to discuss a novel approach that treats both ES and QES systems on the same footing 7 . It is based on the so-called prepotential together with the Bethe ansatz equations. 
Since QES systems are less familiar to most people, I shall give a very brief introduction to QES theory before the prepotential approach is discussed.

\section{A brief introduction to QES theory}

The essence of quasi-exact solvability is most easily explained as follows 4 . It is well known that any Hamiltonian $H$ can be represented as an infinite-dimensional Hermitian matrix

$$
H=\left[\begin{array}{ccccc}
H_{00} & H_{01} & \cdots & H_{0 N} & \cdots \\
H_{10} & H_{11} & \cdots & H_{1 N} & \cdots \\
\cdots & \cdots & \cdots & \cdots & \cdots \\
H_{N 0} & H_{N 1} & \cdots & H_{N N} & \cdots \\
\cdots & \cdots & \cdots & \cdots & \cdots
\end{array}\right]
$$

where the elements $H_{n m}=\left\langle\psi_{n}|H| \psi_{m}\right\rangle$ depend on the choice of a complete set of orthonormal functions $\psi_{n}$ forming a basis of the Hilbert space. The solution of the spectral problem then reduces to a diagonalization of matrix $\left\{H_{n m}\right\}$. Unfortunately, contrary to the case of finite matrices, there is no general algebraic rules that would allow one to diagonalize the infinite-dimensional $H$.

If the matrix $\left\{H_{n m}\right\}$ is very specific so that it can be reduced to the diagonal form with the aid of an algebraic process, then the quantal system is exactly solvable.

$$
H=\left[\begin{array}{cccccc}
H_{00} & 0 & 0 & 0 & 0 & \cdots \\
0 & H_{11} & 0 & 0 & 0 & \cdots \\
0 & 0 & H_{22} & 0 & 0 & \cdots \\
0 & 0 & 0 & H_{33} & 0 & \cdots \\
\cdots & \cdots & \cdots & \cdots & \cdots & \cdots
\end{array}\right]
$$

The most well-known example is the harmonic oscillator.

Suppose a Hamiltonian $H$ is reducible to a block form

$$
H=\left[\begin{array}{cccccccc}
H_{00} & H_{01} & \ldots & H_{0 N} & 0 & 0 & \ldots & 0 \\
H_{10} & H_{11} & \cdots & H_{1 N} & 0 & 0 & \ldots & 0 \\
\ldots & \ldots & \ldots & \ldots & \ldots & \ldots & \ldots & \ldots \\
H_{N 0} & H_{N 1} & \ldots & H_{N N} & 0 & 0 & \ldots & 0 \\
0 & 0 & \ldots & 0 & * & * & \ldots & \ldots \\
0 & 0 & \ldots & 0 & * & * & \ldots & \ldots \\
\ldots & \ldots & \ldots & \ldots & * & * & \ldots & \ldots \\
0 & 0 & \ldots & 0 & * & * & \ldots & \ldots \\
\ldots & \ldots & \ldots & \ldots & * & * & \ldots & \ldots
\end{array}\right],
$$

where the block in the upper left corner is an $(N+1) \times(N+1)$ Hermitian matrix, and the block with asterisks is an infinite matrix with non-vanishing elements. The upper left block can be diagonalized without touching the infinite one. This means that one can determine only a part of the spectrum of $H$ with $N+1$ eigenvalues and eigenfunctions. This system is called QES. 
QES models are not simply mathematical constructs. Although the first and simplest example of QES model is the sextic oscillator, physical QES systems have been found. For examples, the system of two electrons in a three-dimensional oscillator potential ${ }^{8}$, two-dimensional charged particle in Coulomb and uniform magnetic fields (the Schrödinger, Klein-Gordon, and Dirac cases are discussed in Refs. 9, 10 and 11, respectively), and electron on a lattice in magnetic field (Azbel-Hofstadter problem) 12 .

Usually a QES problem admits a certain underlying Lie algebraic symmetry which is responsible for the quasi-exact solutions. Such underlying symmetry is most easily studied in the Lie-algebraic approach $3 \mid 5$. The essence of this approach is as follows. Consider a Schrödinger equation $H \psi=E \psi$ with Hamiltonian $H=$ $-d^{2} / d x^{2}+V(x)$ and wave function $\phi(x)$. Here $x$ belongs either to the interval $(-\infty, \infty)$ or $[0, \infty)$. Now suppose we make an "imaginary gauge transformation" on the function $\phi: \phi(x)=\tilde{\phi}(x) e^{-W_{0}(x)}$. For physical systems which we are interested in, the phase factor $\exp \left(-W_{0}(x)\right)$ is responsible for the asymptotic behaviors of the wave function so as to ensure normalizability. The function $\tilde{\phi}(x)$ satisfies a Schrödinger equation with a gauge transformed Hamiltonian $H_{W}=e^{W_{0}} H e^{-W_{0}}$. Suppose $H_{W}$ can be written as a quadratic combination of the generators $J^{a}$ of some Lie algebra with a finite dimensional representation, i.e.,

$$
H_{W}=\sum c_{a b} J^{a} J^{b}+\sum c_{a} J^{a}+\text { real constant, }
$$

where the constants $c_{a b}$ and $c_{a}$ are usually taken to be real 31516. Then within this finite dimensional Hilbert space the Hamiltonian $H_{W}$ can be diagonalized, and therefore a finite number of eigenstates are solvable. Hence the system described by $H$ is QES. For one-dimensional QES systems the most general Lie algebra is $\operatorname{sl}(2)$. The generators $J^{a}$ of the $s l(2)$ Lie algebra take the differential forms: $J^{+}=$ $z^{2} d_{z}-n z, J^{0}=z d_{z}-n / 2, J^{-}=d_{z}(n=0,1,2, \ldots)$. The variables $x$ and $z$ are related by some function. $n$ is the degree of the eigenfunctions $\tilde{\phi}$, which are polynomials in a $(n+1)$-dimensional Hilbert space with the basis $\left\langle 1, z, z^{2}, \ldots, z^{n}\right\rangle$.

The Lie-algebraic approach to QES models excels in revealing the underlying symmetry of a QES system explicitly. However, solutions of QES states are more directly found in the analytic approach based on the Bethe ansatz equations 4 . In this analytic approach the form of the wave functions containing some parameters are assumed from the very beginning, and these parameters are fitted to make the ansatz compatible with the potential under consideration .

Recently, a different approach to QES models has emerged 13114. In this approach the emphasis shifts from the potential to the so-called prepotential (or superpotential), a concept which plays a fundamental role in supersymmetric quantum mechanics (we note here that the prepotential considered here is the integral of the superpotetial in Refs. 13 and 15). Prepotential has been extensively employed to study classical and quantum integrability in Calogero-Moser systems 16. The merit of this approach is that the form of the potential of the system concerned needs not be assumed from the beginning. All information about the system is contained 
in the prepotential and the solutions, or roots, of the Bethe ansatz equations (in terms of certain transformed coordinates). The prepotential and the roots determine the potential as well as the eigenfunctions and eigenvalues simultaneously. Also, in this approach exact and quasi-exact solvabilities can be treated on the same footing. Furthermore, such approach facilitates extension of the QES theory from the Schrödinger equation to equations for multi-component wave functions $13 \mid 23$. More recently, QES theory was extended to the Fokker-Planck equations also via the prepotential approach 18 .

The emphasis of the works just mentioned was placed on the feasibility and elegance of the prepotential approach. But in these works the forms of the prepotential and the required change of coordinates were either directly adapted from the known ES and QES models, or given as known for the new QES systems. To make the prepotential approach a satisfactory approach one must be able to determine the choice of coordinate transformation and the prepotential from the very beginning. This was recently achieved for certain classes of coordinates and prepotentials 7 . In what follows we shall present the main ideas of this approach, and demonstrate how the simplest ES and QES models could be easily derived.

\section{Prepotential approach}

Suppose $\left.\phi_{0}(x)(-\infty<x<\infty)\right)$ is the ground state, with zero energy, of a Hamiltonian $H_{0}: H_{0} \phi_{0}=0$. By the well known oscillation theorem $\phi_{0}$ is nodeless, and thus can be written as $\phi_{0} \equiv e^{-W_{0}(x)}$, where $W_{0}(x)$ is a regular function of $x$. For the square-integrable $\phi_{0}$, this is the simplest example of quasi-exact solvability. This implies that the potential $V_{0}$ is completely determined by $W_{0}: V_{0}={W_{0}^{\prime}}^{2}-W_{0}^{\prime \prime}$, and consequently, the Hamiltonian is factorizable (we adopt the unit system in which $\hbar$ and the mass $m$ of the particle are such that $\hbar=2 m=1$ ):

$$
H_{0}=\left(-\frac{d}{d x}+W_{0}^{\prime}\right)\left(\frac{d}{d x}+W_{0}^{\prime}\right) .
$$

We shall call $W_{0}(x)$ the zero-th order prepotential.

Consider now a wave function $\phi_{N}(N \geq 0)$ which is related to $\phi_{0}$ of $H_{0}$ by $\phi_{N}=\phi_{0} \tilde{\phi}_{N}$, where

$$
\tilde{\phi}_{N}=\left(z-z_{1}\right)\left(z-z_{2}\right) \cdots\left(z-z_{N}\right), \quad \tilde{\phi}_{0} \equiv 1 .
$$

Here $z=z(x)$ is some function of $x$. In taking the form of $\tilde{\phi}_{N}$ in Eq. (6) I have assumed that the only singularities of the system are $z= \pm \infty$. Other situations are discussed in Ref. 7. The function $\tilde{\phi}_{N}$ is a polynomial in an $(N+1)$-dimensional Hilbert space with the basis $\left\langle 1, z, z^{2}, \ldots, z^{N}\right\rangle$. One can rewrite $\phi_{N}$ as

$$
\phi_{N}=\exp \left(-W_{N}\left(x,\left\{z_{k}\right\}\right)\right),
$$

with the $N$-th order prepotential $W_{N}$ being defined by

$$
W_{N}\left(x,\left\{z_{k}\right\}\right)=W_{0}(x)-\sum_{k=1}^{N} \ln \left|z(x)-z_{k}\right| .
$$


Operating on $\phi_{N}$ by the operator $-d^{2} / d x^{2}$ results in a Schrödinger equation $H_{N} \phi_{N}=0$, where

$$
\begin{aligned}
H_{N} & =-\frac{d^{2}}{d x^{2}}+V_{N}, \\
V_{N} & \equiv W_{N}^{\prime 2}-W_{N}^{\prime \prime} .
\end{aligned}
$$

From Eq. (8), $V_{N}$ has the form $V_{N}=V_{0}+\Delta V_{N}$, where $V_{0}=W_{0}^{\prime 2}-W_{0}^{\prime \prime}$, and

$$
\Delta V_{N} \equiv-2\left(W_{0}^{\prime} z^{\prime}-\frac{z^{\prime \prime}}{2}\right) \sum_{k=1}^{N} \frac{1}{z-z_{k}}+\sum_{\substack{k, l \\ k \neq l}} \frac{z^{\prime 2}}{\left(z-z_{k}\right)\left(z-z_{l}\right)} .
$$

Here the prime denotes derivative w.r.t. the variable $x$.

$\Delta V_{N}$ is generally a meromorphic function of $z$ with at most simple poles. Let us demand that the residues of the simple poles, $z_{k}, k=1, \ldots, N$ should all vanish. This will result in a set of algebraic equations which the parameters $\left\{z_{k}\right\}$ must satisfy. These equations are called the Bethe ansatz equations for $\left\{z_{k}\right\}$. With $\left\{z_{k}\right\}$ satisfying the Bethe ansatz equations, $\Delta V_{N}$ will have no simple poles at $\left\{z_{k}\right\}$ but it still generally depends on $\left\{z_{k}\right\}$. Thus the form of $V_{N}$ is determined by the choice of $z^{\prime 2}$ and $W_{0}(x)$.

In what follows we would like to demonstrate that the choice of $z^{\prime 2}$ and $W_{0}^{\prime} z^{\prime}$ determine the nature of solvability of the quantal system. We shall restrict our consideration only to those cases where $W_{0}^{\prime} z^{\prime}=P_{m}(z), z^{\prime 2}=Q_{n}(z)$ and $z^{\prime \prime}=R_{l}(z)$ are polynomials in $z$ of degree $m, n$ and $l$, respectively. Of course, $Q_{n}(z)$ and $R_{l}(z)$ are not independent. In fact, from $2 z^{\prime \prime}=d z^{\prime 2} / d z$ we have $l=n-1$ and

$$
z^{\prime \prime}=R_{n-1}(z)=\frac{1}{2} \frac{d Q_{n}(z)}{d z} .
$$

Consequently, the variables $x$ and $z$ are related by (we assume $z(x)$ is invertible for practical purposes)

$$
x(z)= \pm \int^{z} \frac{d z}{\sqrt{Q_{n}(z)}},
$$

and the prepotential $W_{0}(x)$ is determined as

$$
W_{0}(x)=\int^{x} d x\left(\frac{P_{m}(z)}{\sqrt{Q_{n}(z)}}\right)_{z=z(x)} .
$$

Eqs. (13) and (14) define the transformation $z(x)$ and the corresponding prepotential $W_{0}(x)$. Thus, $P_{m}(z)$ and $Q_{n}(z)$ determine the quantum system. Of course, the choice of $P_{m}$ and $Q_{n}$ must be such that $W_{0}$ derived from (14) must ensure normalizability of $\phi_{0}=\exp \left(-W_{0}\right)$.

Now depending on the degrees of the polynomials $P_{m}$ and $Q_{n}$, we have the following situations: 
(i) if $\max \{m, n-1\} \leq 1$, then in $V_{N}(x)$ the parameter $N$ and the roots $z_{k}$ 's will only appear as an additive constant and not in any term involving powers of $z$. Such system is then exactly solvable;

(ii) if $\max \{m, n-1\}=2$, then $N$ will appear in the first power term in $z$, but $z_{k}$ 's only in an additive term. This system then belongs to the so-called type 1 QES system defined in Ref. 3, i.e., for each $N \geq 0, V_{N}$ admits $N+1$ solvable states with the eigenvalues being given by the $N+1$ sets of roots $z_{k}$ 's. This is the main type of QES systems considered in the literature;

(iii) if $\min \{m, n-1\} \geq 3$, then not only $N$ but also $z_{k}$ 's will appear in terms involving powers of $z$. This means that for each $N \geq 0$, there are $N+1$ different potentials $V_{N}$, differing in several parameters in terms involving powers of $z$, have the same eigenvalue (when the additive constant, or the zero point, is appropriately adjusted). When $z_{k}$ 's appear only in the first power term in $z$, such systems are called type 2 QES systems in Ref. 3 . We see that QES models of higher types are possible.

These general situations are considered for cases where $z^{\prime 2}=Q_{2}(z)$ are quadratic in $z$ (this includes $Q_{1}$ and $Q_{0}$ as special cases if some of the coefficients vanish) in Ref. 7. This choice of $z^{\prime 2}$ covers most of the known ES shape-invariant potentials in Ref. 15 and the $s l(2)$-based QES systems in Ref. 3, and a new one discussed in Refs. 14 and 18. Such coordinates are called "sinusoidal coordinates", which include quadratic polynomials, trigonometric, hyperbolic, and exponential types. The connection of sinusoidal coordinates with ES theory has been extensively discussed in Ref. 19. For simplicity, in this talk I shall only discuss cases in which $z^{\prime 2}$ is linear in $z$, i.e., $z^{\prime 2}=Q_{1}(z)=q_{1} z+q_{0}$, where $q_{1}$ and $q_{0}$ are real constants.

With this choice of $z^{\prime 2}$, we have

$$
V_{N}={W_{0}^{\prime}}^{2}-W_{0}^{\prime \prime}-2 \sum_{k=1}^{N} \frac{1}{z-z_{k}}\left\{P_{m}(z)-\frac{q_{1}}{4}-\sum_{l \neq k} \frac{Q_{1}\left(z_{k}\right)}{z_{k}-z_{l}}\right\} .
$$

In deriving Eq. (15) use has been made of the following identities:

$$
\begin{aligned}
& \sum_{\substack{k, l=1 \\
k \neq l}}^{N} \frac{1}{\left(z-z_{k}\right)\left(z-z_{l}\right)}=2 \sum_{\substack{k, l=1 \\
k \neq l}}^{N} \frac{1}{z-z_{k}}\left(\frac{1}{z_{k}-z_{l}}\right), \\
& \sum_{\substack{k, l=1 \\
k \neq l}}^{N} \frac{z}{\left(z-z_{k}\right)\left(z-z_{l}\right)}=2 \sum_{\substack{k, l=1 \\
k \neq l}}^{N} \frac{1}{z-z_{k}}\left(\frac{z_{k}}{z_{k}-z_{l}}\right) .
\end{aligned}
$$

Demanding the residues at $z_{k}$ 's vanish gives the set of Bethe ansatz equations

$$
P_{m}\left(z_{k}\right)-\frac{q_{1}}{4}-\sum_{l \neq k} \frac{Q_{1}\left(z_{k}\right)}{z_{k}-z_{l}}=0, \quad k=1,2, \ldots, N .
$$

Putting back the set of roots $z_{k}$ into Eq. (15), we obtain a potential $V_{N}(x)$ without simple poles. The degree of $P_{m}(z)$ determines the nature of the solvability of the 
system, namely, for $m=1,2,3, \ldots$, the system is, respectively, ES, type 1 QES, type 2 QES, and higher types QES, as discussed generally in the last section.

To proceed further, we must specify $P_{m}(z)$. Below I shall discuss cases with $m \leq 2$, i.e., only ES and type 1 QES models.

\section{Examples}

Let us take $Q_{1}(z)=4 A z+q_{0}\left(q_{1}=4 A\right)$, where $A, q_{0}$ are real constants. This implies $z^{\prime \prime}(x)=R_{0}(z)=2 A$. Hence, the general solution of $z(x)$ is a quadratic form of $x$ :

$$
z(x)=A x^{2}+B x+C, A, B, C: \text { real constants. }
$$

It is easily checked that $q_{0}$ is related to $A, B$ and $C$ through $z^{\prime 2}=4 A z+B^{2}-4 A C$.

We now show how some ES and QES models can be constructed in the prepotential approach by taking different values of $m$.

\subsection{Exactly solvable cases: $m=1$}

Suppose $m=1$ so that $P_{1}(z)=A_{1} z+A_{0}\left(A_{1}, A_{0}\right.$ real $)$. By writing $P_{1}=A_{1}(z-$ $\left.z_{k}\right)+A_{1} z_{k}+A_{0}$, one obtains from Eq. (15) that

$$
\begin{aligned}
\Delta V_{N} & =-2 A_{1} \sum_{k=1}^{N} 1-2 \sum_{k=1}^{N} \frac{1}{z-z_{k}}\left\{P_{1}\left(z_{k}\right)-A-\sum_{l \neq k} \frac{Q_{1}\left(z_{k}\right)}{z_{k}-z_{l}}\right\} \\
& =-2 A_{1} N .
\end{aligned}
$$

The last term in braces in Eq. (20) vanishes when $z_{k}$ 's satisfy the Bethe ansatz equations (18). Now $N$ only appears as a parameter in an additive term, and not in terms involving powers of $z$ in $\Delta V_{N}$. The roots $z_{k}$ 's do not appear at all. The additive term can be treated as the eigenvalue. The Schrödinger equation reads

$$
\left(-\frac{d^{2}}{d x^{2}}+W_{0}^{\prime 2}-W_{0}^{\prime \prime}\right) e^{-W_{N}}=2 A_{1} N e^{-W_{N}} .
$$

We see that the potential $W_{0}^{\prime 2}-W_{0}^{\prime \prime}$ is ES: by varying $N$, one obtains all the eigenvalues $2 A_{1} N$ and the eigenfunctions $\phi_{N}=\exp \left(-W_{N}\right)$.

As an example, let us take $Q_{1}(z)=1$ and $P_{1}(z)=b z$. A particular solution of $z$ is $z(x)=x$. From Eq. (14) one gets

$$
W_{0}(x)=\int^{x} \frac{b x}{1} d x=\frac{b x^{2}}{2}+\text { const. }
$$

The integration constant is to be determined by normalization of the wave function. For $\phi_{0}=\exp \left(-W_{0}\right)$ to be square-integrable, one must assume $b>0$. The BAE (18) are:

$$
b x_{k}-\sum_{l \neq k} \frac{1}{x_{k}-x_{l}}=0, \quad k=1, \ldots, N,
$$


and the potential is

$$
V_{0}=b^{2} x^{2}-b, \quad \Delta V_{N}=-2 N b .
$$

This leads to the Schrödinger equation:

$$
\left(-\frac{d^{2}}{d x^{2}}+b^{2} x^{2}\right) e^{-W_{N}}=b(2 N+1) e^{-W_{N}} .
$$

This system is just the well-known simple harmonic oscillator.

We note here that by rescaling $\sqrt{b} x_{k} \rightarrow x_{k}$, Eq. (23) will have $b=1$. The resulted equations are the equations that determine the zeros of the Hermite polynomials $H_{N}(x)$ as found by Stieltjes 20|21|16. Hence we have reproduced the well known wave functions for the harmonic oscillator, namely, $\phi_{N}=\exp \left(-W_{N}\right) \sim$ $\exp \left(-b x^{2} / 2\right) H_{N}(\sqrt{b} x)$.

\subsection{Type 1 quasi-exactly solvable cases: $m=2$}

Next we consider $P_{2}(z)=A_{2} z^{2}+A_{1} z+A_{0}$. By a similar argument we obtain

$$
\Delta V_{N}=-2 A_{2} N z-2 A_{2} \sum_{k=1}^{N} z_{k}-2 A_{1} N .
$$

The Schrödinger can be written as

$$
\left(-\frac{d^{2}}{d x^{2}}+W_{0}^{\prime 2}-W_{0}^{\prime \prime}-2 A_{2} N z\right) e^{-W_{N}}=2\left(A_{2} \sum_{k=1}^{N} z_{k}+A_{1} N\right) e^{-W_{N}} .
$$

Unlike the previous case, now $N$ not only appears in an additive constant term but also in the term with $z$, and the set of roots $z_{k}$ 's appear in the additive term. This system is the so-called type 1 QES models. Type 1 QES models classified as class VI in Ref. 3 belong to this category.

A well-known example is the sextic oscillator, the simplest QES model of this type 1, which is termed as class VI in Ref. 3. In our prepotential approach, this system is defined by $z(x)=x^{2}$ and $P_{2}(z)=2\left(a z^{2}+b z\right)$. Then $Q_{1}(z)=4 z$, and

$$
W_{0}(x)=\int^{x} \frac{a x^{4}+b x^{2}}{\sqrt{x^{2}}} d x=\frac{1}{4} a x^{4}+\frac{1}{2} b x^{2}+\text { const. }
$$

Here $a>0$ to ensure square-integrability of the wave function. The BAE (18) are:

$$
2 a z_{k}^{2}+2 b z_{k}-1-4 \sum_{l \neq k} \frac{z_{k}}{z_{k}-z_{l}}=0, \quad k=1, \ldots, N,
$$

and the potential is

$$
V_{N}=a^{2} x^{6}+2 a b x^{4}+\left[b^{2}-(4 N+3) a\right] x^{2}-4 a \sum_{k} z_{k}-(4 N+1) b .
$$

This is just the well-known QES sextic oscillator first discovered in Ref. 1.

These two examples show how simply and directly the two most classic representatives of ES and QES models are derived in the prepotential approach. Other 
ES and QES models can be constructed in a similar way. Particularly, we can consider cases where $z^{\prime 2}(x)=Q_{2}(z)$ with $q_{2} \neq 0$. Here $z(x)$ is again some sinusoidal coordinates, which include the exponential, hyperbolic and trigonometric functions. By taking appropriate $P_{m}(z)$, one can reconstruct class I, II and X QES models in Ref. 3, and some of the ES models listed in Ref. 15, namely, the Morse potential, the Scarf I and II potentials, and the Pöschl-Teller potential 7 .

\section{Non-Hermitian potentials with real spectra}

Now we turn to a different kind of spectral problem. About ten years ago, it was noted that with properly defined boundary conditions the spectrum of the nonHermitian Hamiltonian $H=p^{2}+x^{2}(i x)^{\nu}(\nu \geq 0)$ is real and positive 22. It is believed that the reality of the spectrum of this Hamiltonian is a consequence of its unbroken $\mathcal{P} \mathcal{T}$ symmetry. This discovery immediately sparked great interest in searching for new systems with such properties, and in understanding the reasons behind the reality of their eigenvalues (for review, see e.g. Ref. 23).

Soon after the appearance of Ref. 22, it was found that, by allowing nonHermitian $\mathcal{P} \mathcal{T}$-symmetric Hamiltonians, a QES polynomial potential can be quartic in its variable 24 . Previously, it was believed the lowest-degree one-dimensional QES polynomial potential is sextic, namely, that presented in Sect. 4. Now let us demonstrate how simply the Hamiltonian discovered in Ref. 24 is derived in our approach if we allow $P_{m}(z)$, or equivalently $W_{0}(x)$, to be complex.

\section{1. $\mathcal{P} \mathcal{T}$-symmetric quartic case}

We take $z=x$ (i.e. $Q_{0}=1$ ), and $P_{2}(x)=a x^{2}+b x+c$ for type 1 QES system. Eq. (14) then gives $W_{0}(x)=a x^{3} / 3+b x^{2} / 2+c x$. It is obvious that, with $a, b$ and $c$ real, the wave functions are not normalizable as the factor $\exp \left(-W_{0}\right)$ does not converge on the whole line. Hence a QES Hamiltonian with quartic polynomial potential is impossible with real coefficients in $W_{0}$.

What if we allow $P_{2}(x)$ to be complex? Suppose we take $a=i \alpha, b=\beta$ and $c=i \gamma$, where $\alpha, \beta>0$ and $\gamma$ are real. Obviously $\exp \left(-W_{0}\right)$ is now square-integrable on the whole line for $b>0$. From Eqs. (15) and (18) we obtain straightforwardly the type 1 QES potential

$$
\begin{aligned}
V_{N}(x)= & -\alpha^{2} x^{4}+2 i \alpha \beta x^{3}+\left(\beta^{2}-2 \alpha \gamma\right) x^{2}-2 i[(N+1) \alpha-\beta \gamma] x \\
& -\left[\gamma^{2}+\beta+2 i \alpha \sum_{k} x_{k}\right],
\end{aligned}
$$

with the BAE

$$
i \alpha x_{k}^{2}+\beta x_{k}+i \gamma-\sum_{l \neq k} \frac{1}{x_{k}-x_{l}}=0, \quad k=1,2, \ldots, N .
$$

$V_{N}$ given by Eq. (31) is precisely the $\mathcal{P} \mathcal{T}$-symmetric QES quartic potential obtained in Ref. 24] (in the notation of Ref. 24, the potential has parameters $\alpha=1, \beta=$ 
$a, \gamma=b$, and $N+1=J)$. In Ref. 24 it was shown that this $\mathcal{P} \mathcal{T}$-symmetric Hamiltonian is also related to the Lie-algebra $s l(2, C)$. It is nice to see that in our prepotential approach this potential can be so simply and directly derived, without any knowledge of its underlying symmetries.

The properties of this system have been fully described in Ref. 24, so we will not attempt a full discussion of it. Let us just consider the two simplest cases with $N=0$ and $N=1$.

For $N=0$, the potential is just $V_{0}$ with only one solvable state corresponding to energy $E=0$. For $N=1$, there are two solvable states. From Eq. (31) we can take the potential to be

$$
V_{1}=-\alpha^{2} x^{4}+2 i \alpha \beta x^{3}+\left(\beta^{2}-2 \alpha \gamma\right) x^{2}-2 i(2 \alpha-\beta \gamma) x
$$

with eigenvalues $\gamma^{2}+\beta+2 i \alpha x_{1}$. The roots $x_{1}$ satisfy the BAE (32): $i \alpha x_{1}^{2}+\beta x_{1}+$ $i \gamma=0$. The solutions are: $x_{1}=i\left(\beta \pm \sqrt{\beta^{2}+4 \alpha \gamma}\right) / 2 \alpha$. Hence we see that for $\beta^{2}+4 \alpha \gamma>0$ the non-Hermitian potential $V_{1}$ has two normalizable states with real energies $\gamma^{2} \pm \sqrt{\beta^{2}+4 \alpha \gamma}$. But if $\beta^{2}+4 \alpha \gamma<0$, the energies are complex. This is consistent with the numerical results in Ref. 24. For higher values of $N$ the analysis is more complicated and numerics are needed 24 .

\subsection{Sextic case without $\mathcal{P} \mathcal{T}$ symmetry}

We now consider a second non-Hermitian QES system. Suppose we assume the parameter $b$ in the sextic oscillator (class VI in Ref. 3) in Sect. 4.2 to be purely imaginary: $b=i \beta$, where $\beta$ and $a>0$ are real. The factor $\exp \left(-W_{0}\right)$ is squareintegrable. Now the potential and BAE are:

$$
\begin{gathered}
V_{N}=a^{2} x^{6}+2 i a \beta x^{4}-\left[\beta^{2}+(4 N+3) a\right] x^{2}-4 a \sum_{k} z_{k}-i(4 N+1) \beta, \\
2 a z_{k}^{2}+2 i \beta z_{k}-1-4 \sum_{l \neq k} \frac{z_{k}}{z_{k}-z_{l}}=0, \quad k=1, \ldots, N .
\end{gathered}
$$

Again, this non-Hermitian system could have real eigenvalues. Trivially for $N=0$ the potential $V_{0}$ admits one normalizable eigenstate with real energy $E=0$. For $N=2$, the solutions of Eq. (35) are: $z_{1}=\left(-i \beta \pm \sqrt{2 a-\beta^{2}}\right) / 2 a$. Then Eq. (34) becomes

$$
V_{1}=a^{2} x^{6}+2 i a \beta x^{4}-\left(\beta^{2}+7 a\right) x^{2}-3 i \beta \pm 2 \sqrt{2 a-\beta^{2}} .
$$

Hence the non-Hermitian potential defined by the first four terms in Eq. (36) has two real eigenvalues $E= \pm 2 \sqrt{2 a-\beta^{2}}$ if $\beta^{2}<2 a$. The energies become complex if $\beta^{2}>2 a$. Like the quartic case just discussed, for higher values of $N$ the analysis of its spectrum requires the aid of numerics. Let us emphasize that this systems is not $\mathcal{P} \mathcal{T}$-symmetric.

We note here that the same analysis can be applied to class I and VII QES systems in Ref. 3 to get other non-Hermitian QES systems with real energies. 


\section{Summary}

I hope I had given you a general idea of how simple and elegant the prepotential approach to ES and QES models is. In this talk I have limited myself to discussing our approach only to systems defined on the full line owing to time limitation. Our approach has been extended to ES and QES systems defined on half-line and on finite intervals 7 . It can be easily extended to the construction of exactly and quasiexactly solvable Dirac, Pauli 13 , Fokker-Planck equations 18 , and to quasinormal modes, which are eigen-modes with complex energies of a Hermitian Hamiltonian 25.

Of course, what I have presented here is quite preliminary. Our approach could be further developed. Firstly, so far we have only considered cases for which $W_{0}^{\prime} z^{\prime}$ and $z^{\prime 2}$ are polynomials in $z$. It is interesting to consider other possibilities for these two defining functions. Secondly, it is extremely interesting to see how our formalism could be extended to many-body systems, such as the renowned CalogeroSutherland-Moser systems. The analogues of Bethe-ansatz equations for these systems are not known yet. Lastly, although some non-Hermitian Hamiltonians can be generated in this approach by making some coefficients in $P_{m}(z)$ complex, as we have shown here, a full treatment of these kind of systems usually requires one to extend the basic variable $x$ to the complex plane $22|23| 24$. Hence a better understanding of the prepotential approach to non-Hermitian Hamiltonians is needed. We hope to report on our progress in these directions in the near future.

\section{Acknowledgments}

I thank R. Sasaki for discussions and for bringing my attention to sinusoidal coordinates. I also thank A. Turbiner for encouragement and stimulating comments on many-body systems. This work is supported in part by the National Science Council (NSC) of the Republic of China under Grant Nos. NSC 96-2112-M-032-007-MY3 and NSC 95-2911-M-032-001-MY2.

\section{References}

1. A.V. Turbiner and A.G. Ushveridze, Phys. Lett. A126, 181 (1987).

2. A.G. Turbiner, Sov. Phys. JETP 67, 230 (1988).

3. A.V. Turbiner, Comm. Math. Phys. 118, 467 (1988).

4. A.G. Ushveridze, Sov. Phys.-Lebedev Inst. Rep. 2 (1988) 50; 54; Quasi-exactly solvable models in quantum mechanics (IOP, Bristol, 1994).

5. N. Kamran and P.J. Olver, J. Math. Anal. Appl. 145, 342 (1990) ; A. González-López, N. Kamran and P.J. Olver, Comm. Math. Phys. 153, 117 (1993).

6. M.A. Shifman and A.V. Turbiner, Comm. Math. Phys. 126, 347 (1989).

7. C.-L. Ho, Prepotential approach to exact and quasi-exact solvabilities, Tamkang University preprint, Nov 2007 (arXiv:0711.3699 [math-ph]).

8. A. Samanta and S.K. Ghosh, Phys. Rev. A42, 1178 (1990); M. Taut, ibid. A48, 3561 (1993).

9. M. Taut, J. Phys. A27, 1045 (1994); ibid. A28, 2081 91995); ibid. A32, 5509 (1999). 
10. V.M. Villalba and R. Pino, Phys. Lett. A238, 49 (1998).

11. C.-L. Ho and V.R. Khalilov, Phys. Rev. A61, 032104 (2000); C.-M. Chiang and C.-L. Ho, J. Math. Phys. 43, 43 (2002); Mod. Phys. Lett. A20, 673 (2005).

12. P. B. Wiegmann and A. V. Zabrodin, Phys. Rev. Lett. 72, 1890 (1994).

13. C.L. Ho and P. Roy, J. Phys. A36, 4617 (2003); Ann. Phys. 312, 161 (2004); C.-L. Ho, ibid. 321, 2170 (2006).

14. R. Sasaki and K. Takasaki, J. Phys. A34, 9533 (2001).

15. F. Cooper, A. Khare and U. Sukhatme, Phys. Rep. 251, 267 (1995).

16. E. Corrigan and R. Sasaki, J.Phys. A35 (2002) 7017; A.J. Bordner, N.S. Manton and R. Sasaki, Prog. Theor. Phys. 103 (2000) 463; S.P. Khastgir, A.J. Pocklington and R. Sasaki, J. Phys. A33 (2000) 9033.

17. Y. Brihaye and P. Kosinski, Mod. Phys. Lett. A13, 1445 (1998) ; Y. Brihaye and A. Nininahazwe, ibid. A20, 1875 (2005).

18. C.-L. Ho and R. Sasaki, Qausi-exactly solvable Fokker-Planck equations, Tamkang preprint and Yukawa Institute (Kyoto Univ.) Report No.Y ITP-07-21, arXiv:0705.0863 v2. (to appear in Ann. Phys.).

19. S. Odake and R. Sasaki, J. Math. Phys. 47, 102102 (2006).

20. T.J. Stieltjies, "Sur quelques théorèmes d'algèbre", Compte Rendus 100, 439 (1885).

21. G. Szegö, Orthogonal Polynomials, Amer. Math. Soc. Colloquium Publications Vol. 23 (Amer. Math. Soc., New York, 1939).

22. C.M. Bender and S. Boettcher, Phys. Rev. Lett. 80, 5234 (1998).

23. C.M. Bender, Contemp.Phys. 46, 277 (2005).

24. C.M. Bender and S. Boettcher, J. Phys. A31, L273 (1998).

25. H.-T.Cho and C.-L. Ho, J. Phys. A40, 1325 (2007). 